\title{
Cerambycidae floricoli (Coleoptera) osservati in aree prative della Valle Scrivia (Genova)
}

\begin{abstract}
Riassunto: Vengono riportati i risultati di uno studio effettuato nel periodo tra giugno e settembre 2018 nella porzione genovese della Valle Scrivia sulle popolazioni di Coleotteri Cerambycidae floricoli, con osservazioni relative alla selezione dei fiori sui quali bottinare e sull'abbondanza in base alle temperature.
\end{abstract}

\begin{abstract}
Floricolous Cerambycidae (Coleoptera) observed in meadow areas of the Scrivia Valley (Genoa).
The results of a study on the floricolous Coleoptera Cerambycidae in the Valle Scrivia (province of Genoa, NW-Italy) are given. Some observation on flower preferences and on the influence of the temperature on their abundance are outlined.
\end{abstract}

Key words: Coleoptera, Cerambycidae, Liguria, Valle Scrivia, food preferences.

\section{INTRODUZIONE}

La presente ricerca si poneva come obiettivi principali una stima della diversità e abbondanza dei Coleotteri Cerambicidi floricoli e una valutazione delle preferenze degli stessi per i fiori di diverse specie botaniche.

Inoltre, si voleva valutare l'influenza della temperatura ambientale sull'abbondanza dei Cerambicidi.

I Cerambycidae, comunemente noti come longicorni, sono insetti diffusi in tutto il mondo, dove hanno colonizzato ogni regione biogeografica ad esclusione di quella antartica. Possono essere osservati dal livello del mare sino ai $4000 \mathrm{~m}$ di altitudine circa, ovunque trovino delle piante idonee per lo sviluppo larvale e per i loro scopi alimentari (Wang, 2017).

Molte specie si nutrono di materia legnosa, spesso di necromassa forestale, penetrando nei tessuti più interni del tronco e delle radici. Ciò è loro consentito, oltre che da un potente apparato masticatore, da una capacità di degradare enzimaticamente molti costituenti del legno attraverso enzimi prodotti dall'insetto stesso (Wang, 2017).

Tra i coleotteri Cerambycidae legati agli ambienti forestali, a livello globale, circa 200 specie sono considerate dannose in ambito agricolo, silvicolturale, oltre che forestale, in particolare specie appartenenti alla sottofamiglia Lamiinae, come ad esempio Anoplophora chinensis Thomson, 1865 (Wang, 2017).

I Coleotteri Cerambycidae sono presenti in Italia con 296 specie (Sama \& Rapuzzi, 2011) sulle circa 600 conosciute per il continente europeo (Gobbi, 2007), mentre a livello globale si contano più di 36.000 specie. Tra le specie presenti sul territorio italiano, si ricordano Cerambyx cerdo Linnaeus, 1758 e Rosalia alpina Linnaeus, 1758, in quanto inseriti negli Allegati II e IV della Direttiva Habitat (92/43/CEE).

Oltre a queste, in Italia sono segnalate anche specie alloctone come Aromia bungii Faldermann (Cerambice dal collo rosso), originaria della Cina e della Corea, rinvenuto per la prima volta in provincia di Milano nel 2013 e la già citata Anoplophora chinensis, segnalata attualmente in Lombardia e Lazio, che attacca anche latifoglie come betulle, carpini, faggi, frassini, pioppi, noccioli.

\section{AREA DI STUDIO}

Lo studio si è svolto interamente in Valle Scrivia, in provincia di Genova e ha visto la scelta di 4 aree prative ad altitudini comprese tra gli $800 \mathrm{~m}$ e i $1300 \mathrm{~m}$ s.l.m.: Monte Porale $(830 \mathrm{~m})$, Alpe di Cassissa-Bric delle Camere (900-1000 m), Passo dell'Incisa-Monte Carmo (1070-1300 m) e Passo della Banca (810 m).

A livello vegetazionale, nel sito Monte Porale si ha la presenza di una vegetazione più arbustiva con dominanza di Juniperus communis (Ginepro comune), Spartium junceum (Ginestra odorosa), Calluna vulgaris (Brugo), con formazioni forestali a Fraxinus sp. e opere di rimboschimento a Pinus nigra (Pino nero) e Quercus petrea (Rovere).

\footnotetext{
*Alessandro Marmugi, Via Maccabelli 15, 16019 Ronco Scrivia (GE), Italia. E-mail: alemarmu.sa93@hotmail.com
} 
Negli altri tre siti si ha la dominanza di castagneti, formazioni a Corylus avellana (Nocciolo), Fraxinus sp. (Frassino), presenza sporadica di orno-ostrieti e formazioni a Quercus cerris (Cerro).

Nei prati oggetto d'indagine, considerando tutto il periodo di studio, le famiglie maggiormente rappresentate nelle fioriture sono risultate essere Apiaceae (tra cui i generi più comuni Anthriscus sp., Daucus sp., Pimpinella sp., a cui si può aggiungere Physospermum cornubiense) e Asteraceae (tra cui le più comuni i generi Achillea sp., Leucanthemum sp., Taraxacum sp., Urospermum sp., a cui si può aggiungere Cichorium intybus).

\section{MATERIALI E METODI}

Lo studio ha previsto, nel periodo tra giugno e settembre 2018, due campionamenti al mese per ogni area individuata, nello specifico durante la terza e quarta settimana del mese, per un totale di 32 uscite in una fascia oraria compresa tra le 11.00 e le 15.00 , per sfruttare i momenti più caldi della giornata e reperire il maggior numero possibili di esemplari.

La raccolta degli esemplari, per evitare un dan- neggiamento dei fiori che potesse condizionare le sessioni successive, è stata effettuata a vista sui fiori presenti lungo un transetto standardizzato di $50 \mathrm{~m}$.

Nel corso di ogni sessione di raccolta in ciascun sito sono state registrate, tramite un termometro portatile, le temperature e annotate le principali specie botaniche in fiore.

Successivamente si è provveduti all'identificazione degli esemplari, avvalendosi dei testi di supporto di Pesarini \& Sabbadini (1994) e Sama (2002).

\section{RISULTATI}

Gli esemplari raccolti sono stati 193, ascrivibili a 15 specie diverse, appartenenti a 3 sottofamiglie (Cerambycinae, Lamiinae, Lepturinae) (Tab. 1); le specie più comuni sono state Stenopterus rufus rufus, Pseudovadonia livida livida e Plagionotus floralis.

Il numero di specie riscontrate non è elevatissimo, in quanto tre delle quattro zone oggetto di studio, dal punto di vista vegetazionale erano piuttosto simili.

Il maggior numero di catture è stato effettuato nei mesi di giugno e di luglio, in particolare in questo

Tab. 1. Specie osservate.

\begin{tabular}{|c|c|c|c|}
\hline Sottofamiglia & Specie & N. individui & Siti di localizzazione \\
\hline \multirow[t]{6}{*}{ Cerambycinae } & Chlorophorus figuratus Scopoli, 1763 & 2 & Alpe di Cassissa; Passo della Banca \\
\hline & Chlorophorus sartor Müller, 1766 & 8 & Alpe di Cassissa; Monte Porale; Passo della Banca \\
\hline & Chlorophorus trifasciatus Fabricius, 1781 & 2 & Alpe di Cassissa; Monte Porale \\
\hline & Deilus fugax Olivier, 1790 & 5 & Passo della Banca \\
\hline & Plagionotus floralis Pallas, 1776 & 17 & Alpe di Cassissa \\
\hline & Stenopterus rufus rufus Linnaeus, 1767 & 82 & $\begin{array}{l}\text { Alpe di Cassissa; Monte Porale; Passo della Banca; } \\
\text { Passo dell'Incisa }\end{array}$ \\
\hline \multirow[t]{2}{*}{ Lamiinae } & Calamobius filum Rossi, 1790 & 5 & $\begin{array}{l}\text { Alpe di Cassissa; Passo della Banca; Passo } \\
\text { dell'Incisa }\end{array}$ \\
\hline & Leiopus nebulos nebulosus Linnaeus, 1758 & 1 & Alpe di Cassissa \\
\hline \multirow[t]{7}{*}{ Lepturinae } & Pachytodes erraticus Dalman, 1817 & 1 & Monte Porale \\
\hline & Paracorymbia fulva de Geer, 1775 & 3 & Alpe di Cassissa; Monte Porale \\
\hline & Pseudovadonia livida livida Fabricius, 1776 & 36 & Passo della Banca; Passo dell'Incisa; Monte Porale \\
\hline & Rutpela maculata maculata Poda, 1761 & 12 & $\begin{array}{l}\text { Alpe di Cassissa; Monte Porale; Passo della Banca; } \\
\text { Passo dell'Incisa }\end{array}$ \\
\hline & Stenurella bifasciata bifasciata Müller, 1776 & 7 & Monte Porale; Passo della Banca \\
\hline & Stenurella melanura Linnaeus, 1758 & 7 & Alpe di Cassissa; Monte Porale \\
\hline & Stenurella nigra Linnaeus, 1758 & 5 & Passo della Banca \\
\hline
\end{tabular}


secondo periodo in quanto le temperature sono state più elevate.

Il picco è stato raggiunto nella quarta settimana di luglio (in concomitanza di una temperatura massima di circa $31^{\circ} \mathrm{C}$ ), in particolare nel sito Alpe di Cassissa: è in questo periodo che sono stati ivi raccolti tutti gli esemplari di Plagionotus floralis.

In agosto e settembre, le temperature hanno iniziato a scendere sotto i $25-26^{\circ} \mathrm{C}$ e anche le fioriture hanno iniziato a scarseggiare, in particolar modo a settembre.

Considerando invece le specie botaniche in fiore, ad Alpe di Cassissa in giugno vi era una notevole fioritura di Taraxacum sp. e Urospermum sp. su cui sono state effettuate catture in particolar modo di Stenopterus rufus rufus, mentre in luglio risultava presente una consistente fioritura di Daucus sp. e Achillea sp. e, pur essendo ancora presenti le fioriture di giugno, queste non sono state quasi prese in considerazione da parte dei cerambicidi, che invece visitavano attivamente $\mathrm{i}$ fiori dei due generi appena sbocciati.

A Passo della Banca, nel mese di agosto è stata osservata una consistente fioritura di Pimpinella sp. (Apiaceae), ma senza riscontrare catture. Tale caso potrebbe essere relazionato alle temperature che durante le uscite di agosto difficilmente superavano i $25-26^{\circ} \mathrm{C}$.

Solo nel sito Monte Porale è stato individuato un esemplare di Pachytodes erraticus Dalman, 1817 che per la Liguria non era stato ancora segnalato (Sama \& Rapuzzi, 2011). È segnalato, tuttavia, nel basso Piemonte, nella zona di Arquata Scrivia, che confina con l'area in oggetto.

\section{CONCLUSIONI}

Le maggiori catture sono state registrate nelle uscite di giugno e luglio, in quanto le temperature sono state più elevate, sino a un picco di $31^{\circ} \mathrm{C}$ nell'area dell'Alpe di Cassissa, nell'ultima settimana di luglio. Ad agosto e settembre le catture effettuate sono state relativamente scarse (1 esemplare di Chlorophorus trifasciatus Fabricius, 1781 nell'area del Monte Porale e 2 esemplari di Rutpela maculata maculata Poda, 1761 nell'area del Passo dell'Incisa).

Nello specifico delle specie trovate, Stenopterus rufus rufus (Cerambycinae) è pressoché dominante in presenza in tutte le aree oggetto di studi, tranne nella zona del Passo dell'Incisa, dove pur essendo presente sino alla terza settimana di luglio, è sempre stato osservato in quantità inferiori rispetto a Pseudovadonia livida livida, che fa la sua comparsa solo nel mese di giugno.
Chlorophorus trifasciatus è stato osservato 2 volte in due diversi siti: una prima volta all'Alpe di Cassissa nell'ultimo periodo di luglio, e una nella zona del Monte Porale nella terza settimana di agosto.

Nel primo caso le temperature superavano i $30^{\circ} \mathrm{C}$, nel secondo le temperature hanno raggiunto un massimo di $25^{\circ} \mathrm{C}$. Potrebbe indicare una maggior sopportazione delle escursioni termiche.

La specie Calamobius filum, legata sia allo stadio larvale sia allo stadio di adulto alle Graminacee, era segnalata, per la Regione Liguria, principalmente nell'estremo ponente e nell'estremo levante (Sama, 1988).

La specie Pachytodes erraticus (Lepturinae) è stato osservato in una sola occasione nell'area del Monte Porale, risultando non ancora segnalata in Liguria (Sama \& Rapuzzi 2011).

Considerando il territorio circostante la Liguria, la specie sopra citata era segnalata nell'area di Arquata Scriva, nel Basso Piemonte, zona al confine con l'area di studio in oggetto, oltre che nei pressi del Colle di Tenda, a cavallo tra le Alpi Liguri e Alpi Marittime (Sama, 1988).

Rifacendosi a ciò che scrive Sama nel 1988, la specie è localmente comune in tutta Italia, con esclusione della Sardegna, con popolazioni osservate nell'arco alpino, in Veneto, nell'area appenninica tra Emilia-Romagna e Toscana, Lazio, Puglia, Basilicata, Calabria e Sicilia.

In base alle osservazioni riportate, il parametro ambientale maggiormente influente sulla attività e sulla presenza dei Cerambicidi pare essere la temperatura. $\mathrm{La}$ presenza di sole pare essere determinante principalmente per il grado di attività, piuttosto che la effettiva presenza degli insetti. In quanto sono state effettuate delle catture anche quando il cielo era parzialmente nuvoloso. Mentre se la copertura era consistente, la presenza dei coleotteri andava a sciamare.

Analizzando le preferenze floricole per l'alimentazione, è stato osservato come Apiaceae e Asteraceae siano le favorite, in particolare il genere Daucus sp. e Pimpinella sp. per le prime e Achillea sp., Leucanthemum sp. e Taraxacum sp. per le seconde. Se contemporaneamente presenti la preferenza verte sulle Asteraceae, nello specifico il genere Achillea.

È stata osservata una preferenza generale per i fiori di color bianco (i generi Achillea sp., Daucus sp. e Pimpinella sp.) rispetto a quelli gialli (Taraxacum sp. e Urospermum sp.) o blu (come Cichorium intybus, il genere Scabiosa o il genere Plantago). 
Solo nell'area dell'Alpe di Cassissa, durante le uscite di giugno, gli esemplari catturati frequentavano i fiori di Taraxacum sp. e di Urospermum sp., in quanto i generi Achillea sp. e Daucus sp. non erano ancora fioriti.

Di seguito vengono riportati i grafici rappresentanti le catture per ogni area oggetto di studio in relazione ai parametri ambientali osservati e ai generi botanici utilizzati per alimentarsi (Figg 1-8): Passo della Banca, Alpe di Cassissa, Passo dell'Incisa, Monte Porale. Inoltre, si riporta un grafico riassuntivo delle catture per ogni sito oggetto di studio (Fig. 9)

I siti Passo della Banca e Alpe di Cassissa presentano caratteristiche vegetazionali e di diversità di specie simile, con quantità di catture superiori rispetto agli altri due siti oggetto di studio. Il sito Passo dell'In- cisa invece presenta il più basso numero di specie riscontrato con solo 4 generi diversi osservati e la specie dominante Pseudovadonia livida livida a discapito di Stenopterus rufus rufus, risultato dominante in tutte le altre aree. Il sito Monte Porale presenta il rinvenimento di 9 specie diverse, paragonabile ai siti Passo della Banca e Alpe di Cassissa, ma con catture minori con solo 21 individui prelevati nel periodo di studio.

\section{RINGRAZIAMENTI}

Ringrazio il Prof. Loris Galli dell'Università di Genova per il supporto e gli insegnamenti ricevuti durante gli anni di studi accademici terminati con la tesi di Laurea, di cui il presente elaborato è un estratto dei risultati ottenuti.

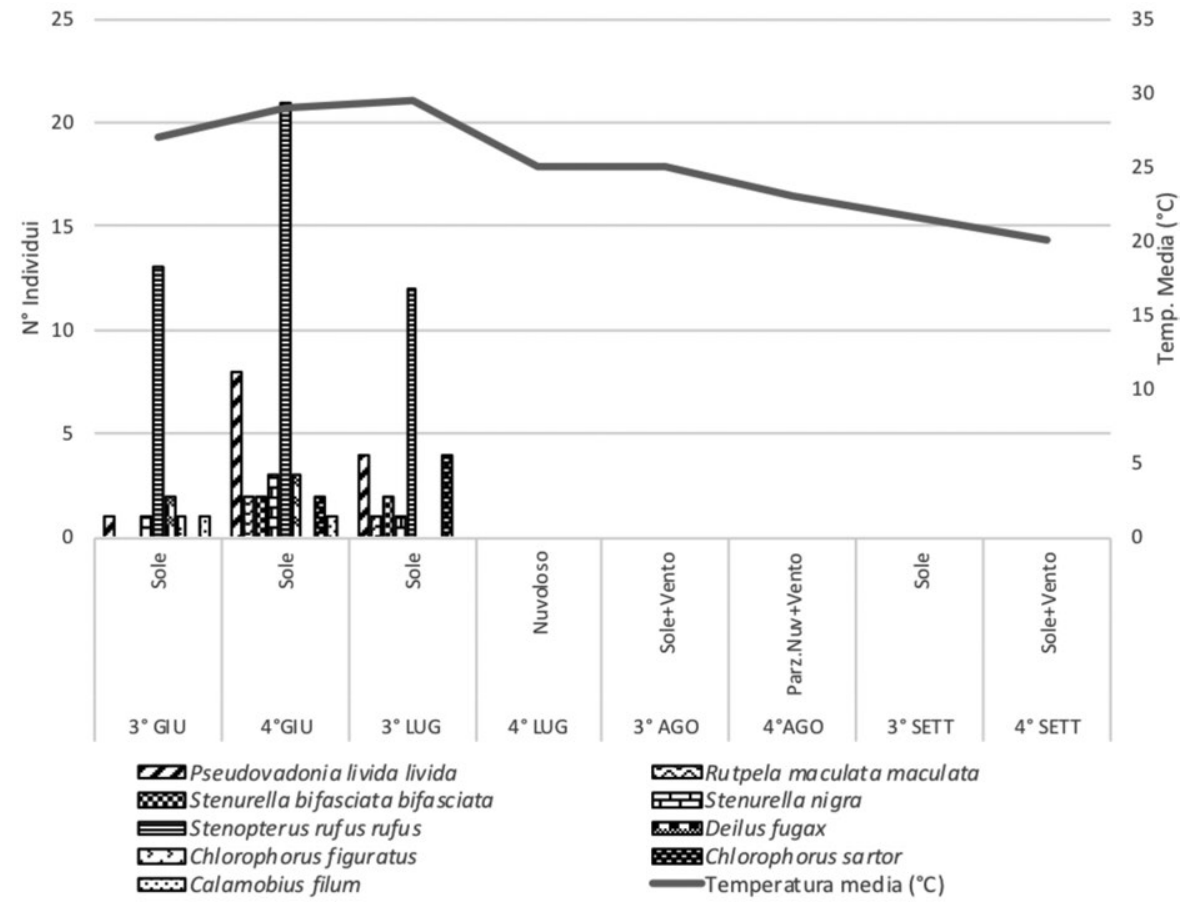

Fig. 1. Relazione tra Cerambicidi e condizioni ambientali nel sito "Passo della Banca". 


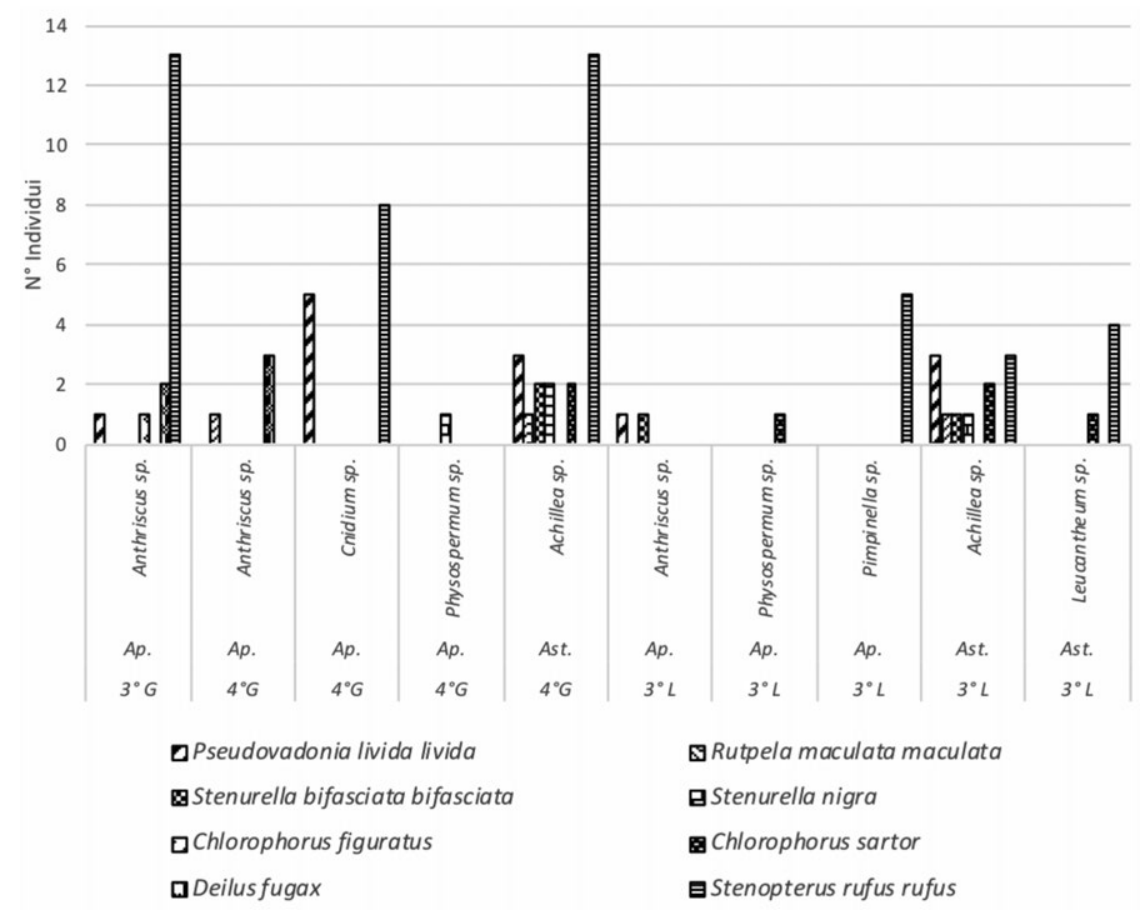

Fig. 2. Relazioni tra Cerambicidi e vegetazione nel sito "Passo della Banca".

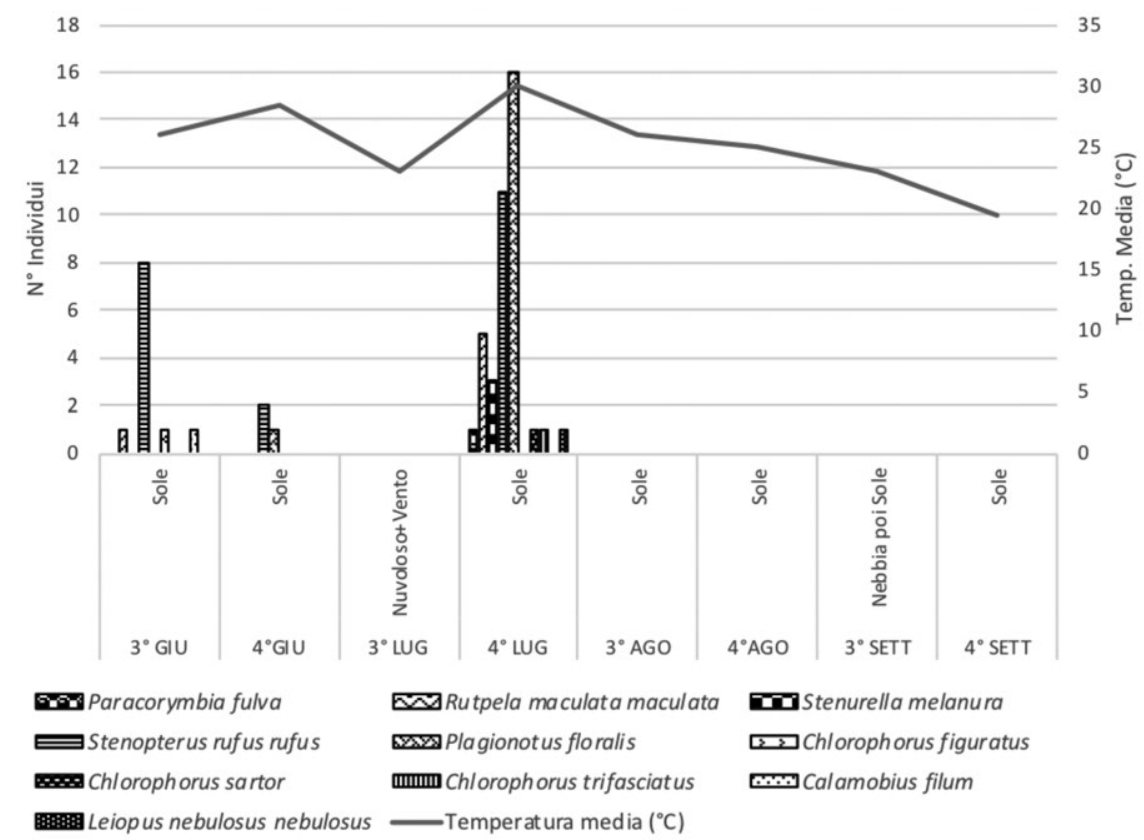

Fig. 3. Relazione tra Cerambicidi e condizioni ambientali nel sito "Alpe di Cassissa". 


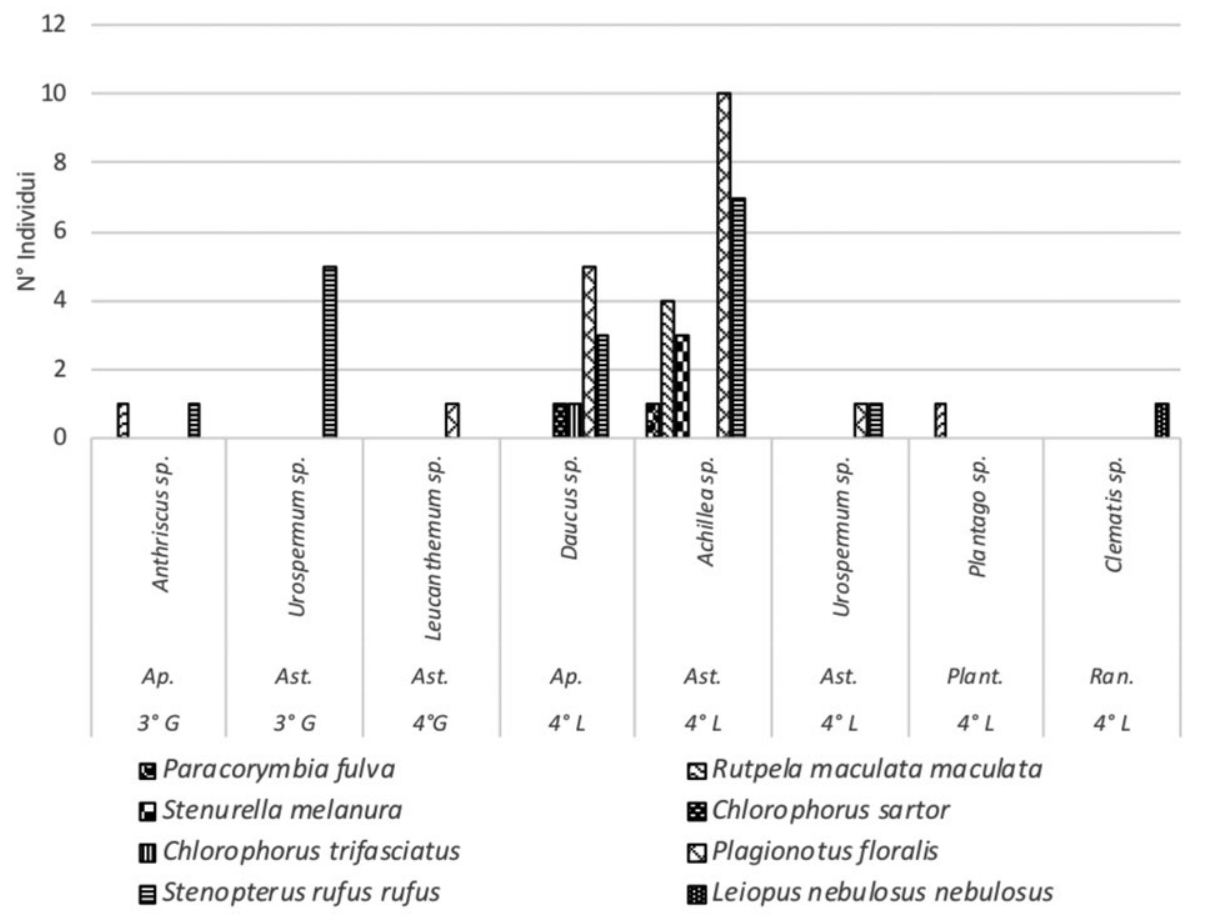

Fig. 4. Relazione tra Cerambicidi e vegetazione nel sito "Alpe di Cassissa".

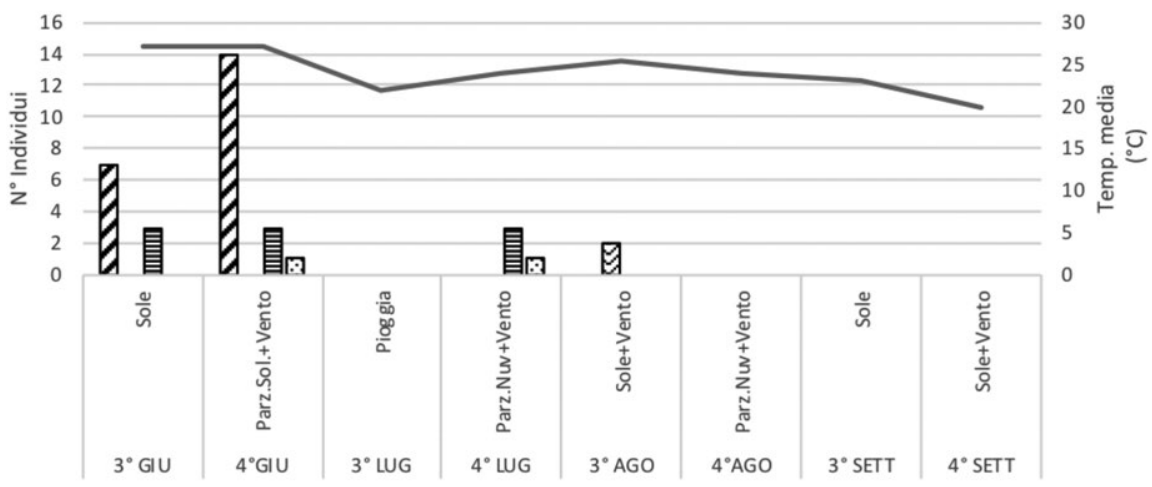

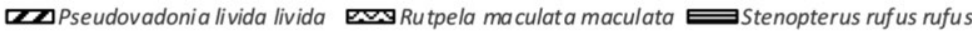
Calamobius filum -Temperatura media $\left({ }^{\circ} \mathrm{C}\right)$

Fig. 5. Relazione tra Cerambicidi e condizioni ambientali nel sito "Passo dell'Incisa". 


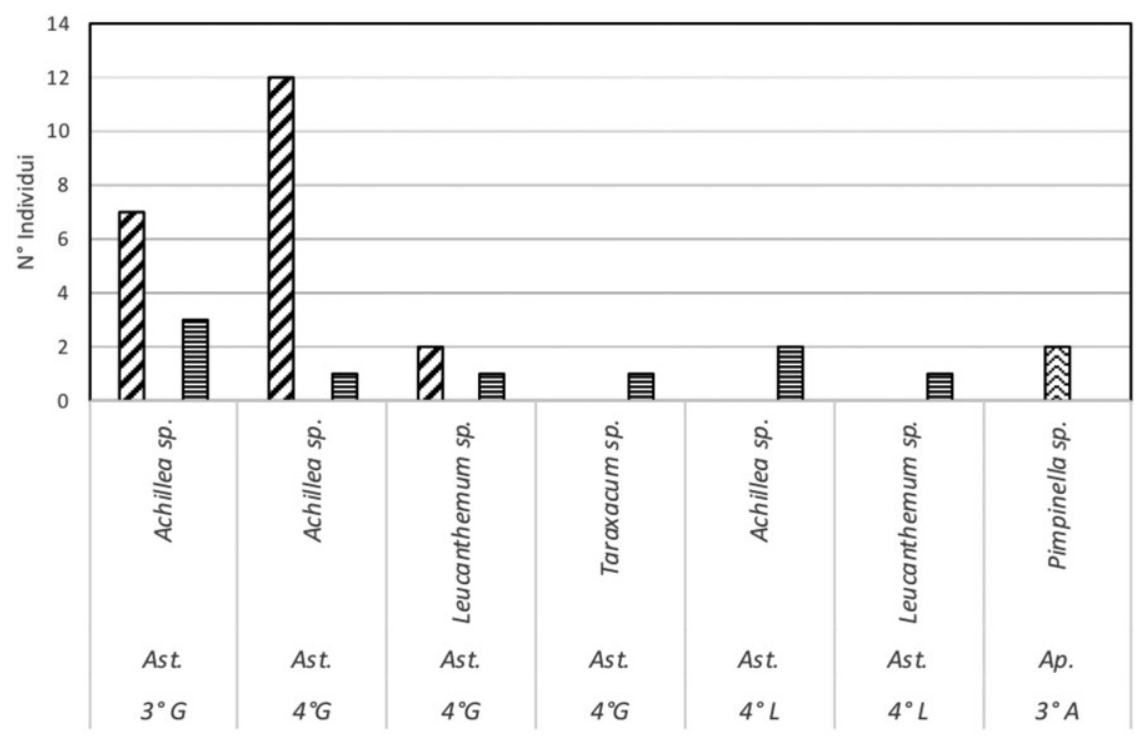

$\square$ Pseudovadonia livida livida $₫$ Rutpela maculata macula ta 目Stenopterus rufus rufus

Fig. 6. Relazione tra Cerambicidi e vegetazione nel sito "Passo dell'Incisa".

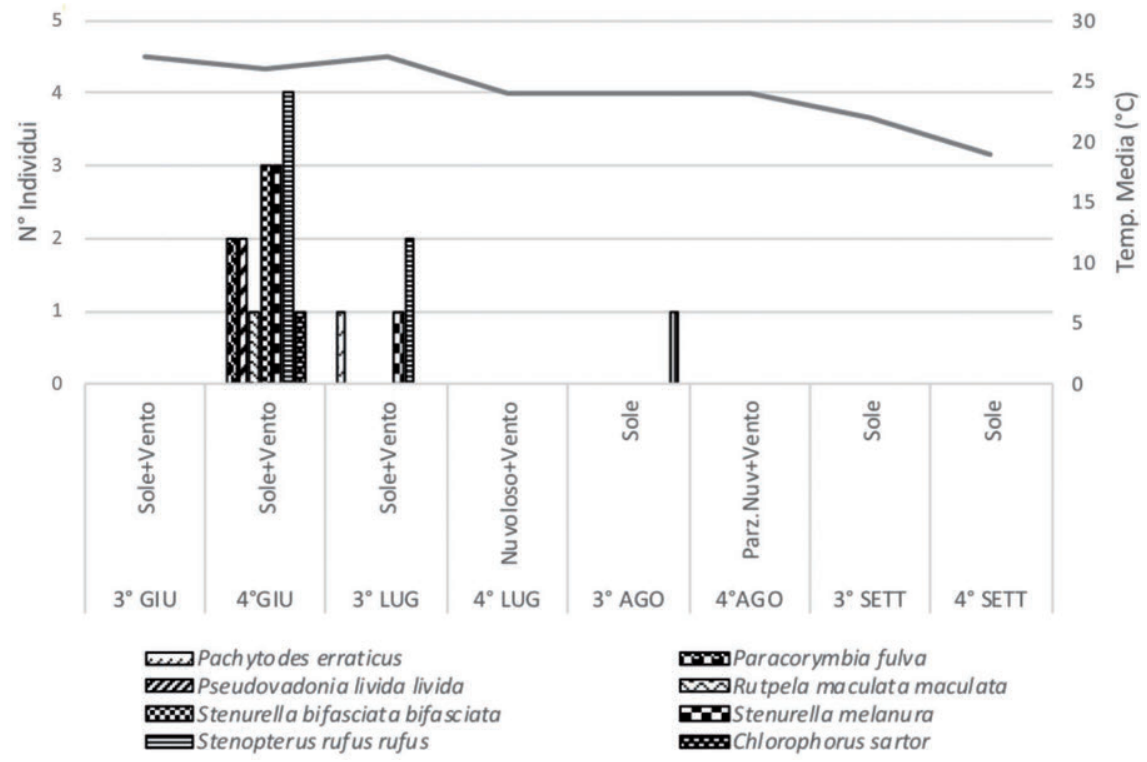

Fig. 7. Relazione tra Cerambicidi e condizioni ambientali nel sito "Monte Porale". 


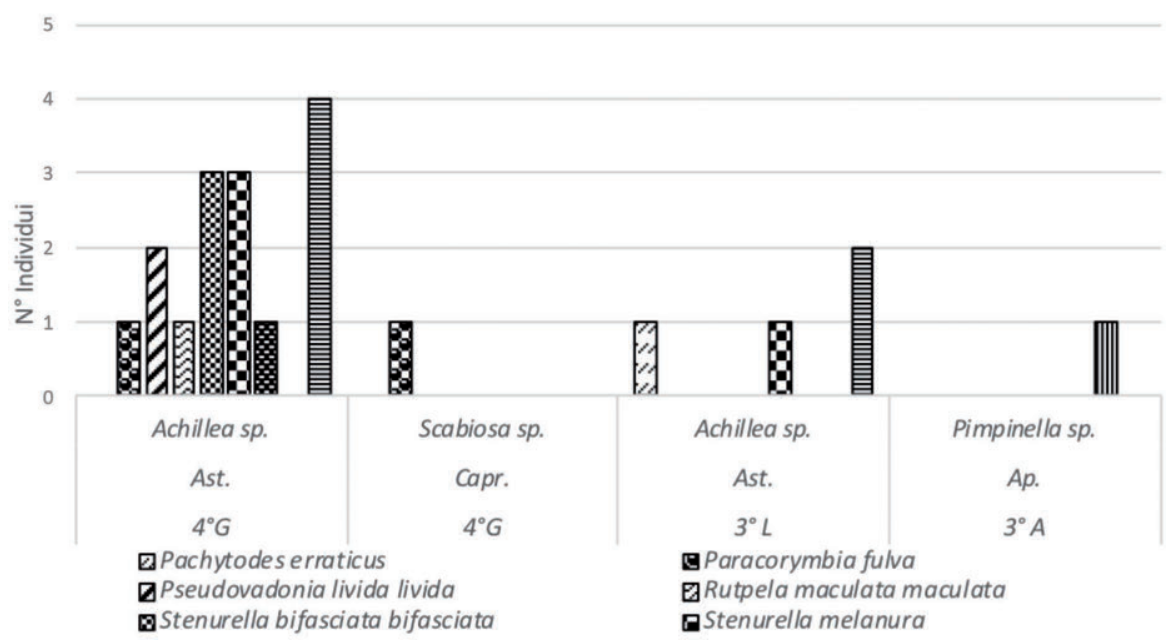

Fig. 8. Relazione tra Cerambicidi e vegetazione nel sito "Monte Porale".

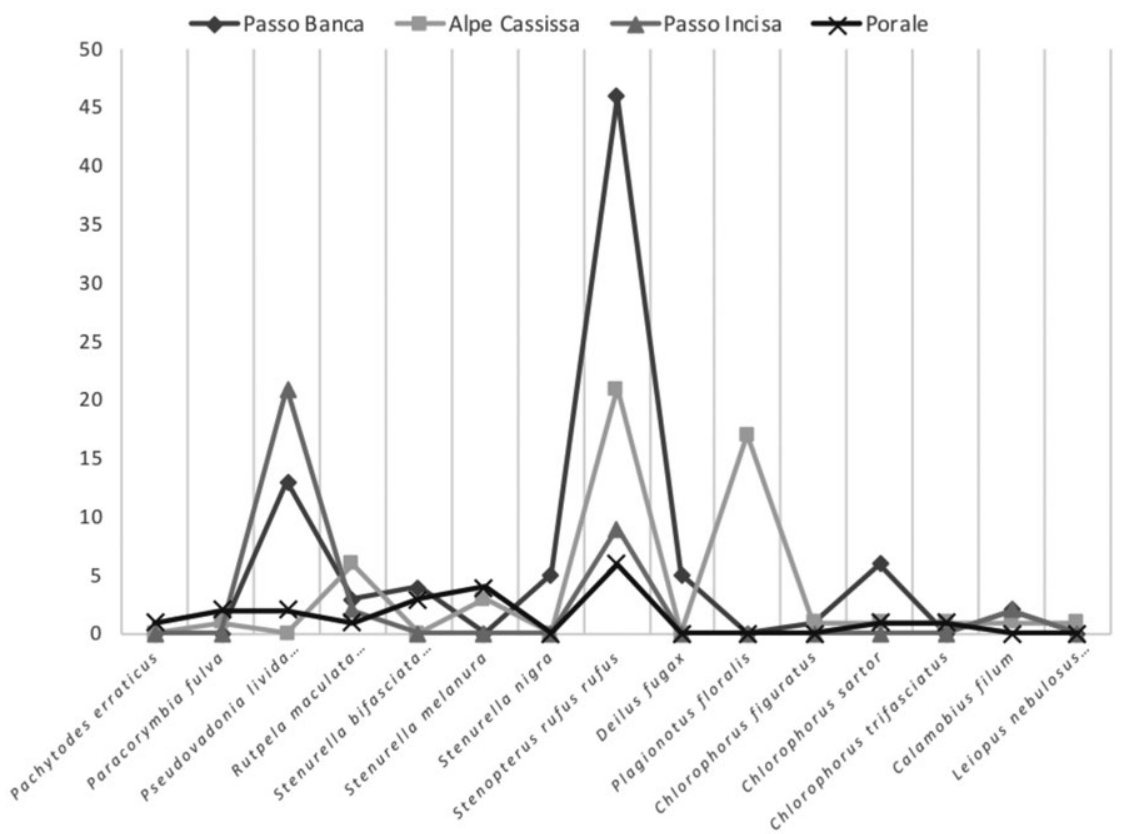

Fig. 9. Catture effettuate per ogni sito oggetto di studio.

\section{BIBLIOGRAFIA}

GobBi M., 2007 - [I Coleotteri Cerambicidi della Val Genova (TN, Trentino): sessant'anni di ricerche], dati non pubblicati. 45 p. https://www.pnab.it/info/area-download/?upf=dl\&id=8009.

Pesarini C., SABbadini A., 1994 - Insetti della Fauna Europea Coleotteri Cerambicidi. Natura, Rivista di Scienze Naturali, 85: 132 p. RApuzZi P., SAMA G., 2011 - Nuova Checklist dei Cerambycidae d'Italia, Quaderni di Studi Naturalistici della Romagna, 32: 121-164. SAma G., 1988 - Fauna d'Italia Coleoptera Cerambycidae, Catalogo topografico e sinonimico. Edizioni Calderini, Bologna. 216 p. SAMA G., 2002 - Atlas of Cerambycidae of Europe and the Mediterranean Area, Vol. 1, Nakladatelstvi Kabourek, Zlin. 173 p. Wang Q., 2017 - Cerambycidae of the World: Biology and Pest Management, 6000 Broken Sound Parkway NW, Suite 300 Boca Raton, (FL), CRC Press. 628 p. 